\title{
DETERMINATION OF SULFUR IN DIESEL USING ATR/ FTIR AND MULTIVARIATE CALIBRATION
}

\author{
Itânia Pinheiro Soares ${ }^{1}$, Thais F. Rezende ${ }^{1}$ e Isabel Cristina P. Fortes ${ }^{1}$ \\ 1- Laboratório de Ensaio de Combustíveis - Depto de Química - Instituto de Ciências Exatas - Universidade Federal de Minas Gerais. Av. \\ Antônio Carlos, 6627 - Campus Pampulha, CEP 31270-901 - Belo Horizonte - Minas Gerais - Brasil
}

\begin{abstract}
The aim of this present work was to provide a more fast, simple and less expensive to analyze sulfur content in diesel samples than by the standard methods currently used. Thus, samples of diesel fuel with sulfur concentrations varying from 400 and $2500 \mathrm{mgkg}^{-1}$ were analyzed by two methodologies: X-ray fluorescence, according to ASTM D4294 and by Fourier transform infrared spectrometry (FTIR). The spectral data obtained from FTIR were used to build multivariate calibration models by partial least squares (PLS). Four models were built in three different ways: 1) a model using the full spectra (665 to $\left.4000 \mathrm{~cm}^{-1}\right)$, 2) two models using some specific spectrum regions and 3) a model with variable selected by classic method of variable selection stepwise. The model obtained by variable selection stepwise and the model built with region spectra between 665 and $856 \mathrm{~cm}^{-1}$ and 1145 and $2717 \mathrm{~cm}^{-1}$ showed better results in the determination of sulfur content.
\end{abstract}

Keywords: PLS, sulfur, ATR/FTIR

\section{Introdução}

O diesel é um combustível fóssil constituído de compostos parafínicos, cicloparafinas, aromáticos e outros em pequena quantidade. A composição do diesel é função da origem do petróleo utilizado para se obter o combustível, da planta de craqueamento utilizada e também do processo de dessulfurização.

A crescente utilização de combustíveis fósseis tem sido responsável por fatores negativos como o aquecimento global e o aumento dos níveis de poluição atmosférica. Os poluentes são uma mistura de gases tóxicos e material particulado. Os gases incluem monoxide de carbon (CO), hidrocarbonetos (HCs), óxidos de nitrogênio $\left(\mathrm{NO}_{\mathrm{x}}\right)$ e óxidos de enxofre $\left(\mathrm{SO}_{\mathrm{x}}\right)$ [1]. Os níveis de enxofre no diesel têm sido identificados como a maior contribuição na poluição do ar atmosférico pelas emissões de particulados e dióxido de enxo- fre de veículos a diesel. Então muitos países têm estabelecido metas para redução das emissões de enxofre no diesel. Nos Estados Unidos, por exemplo, o nível de enxofre aceitável no óleo diesel foi, primeiramente, reduzido de $2000 \mathrm{ppm}$ para 500 ppm pela Clean Air Act ( CAA) e em seguida, para 350 ppm e 50 ppm nos anos 2000 e 2005, respectivamente. Em junho de 2006 iniciou-se a introdução de 15 ppm. O Japão e alguns países da União Européia introduziram diesel no Mercado com níveis de 10 ppm no ano de 2008 [2]. De acordo com a legislação brasileira o teor de enxofre no diesel pode ser determinado por diferentes métodos (ASTM D 1552, D 2622, D 4294 e D 5453). Contudo, algumas dessas metodologias podem apresentar alguns inconvenientes como a necessidade de material de referência a calibração do equipamento com construção de curvas analíticas e problemas de detecção em baixos teores de enxofre. Assim torna-se importante o desenvol- 
vimento de metodologias que superem estas dificuldades e permitam a determinação do teor de enxofre em amostras de diesel de forma simples, rápida e de baixo custo.

A espectroscopia de infravermelho é uma das muitas e diferentes técnicas analíticas que têm sido usadas em análise de combustível. A técnica é não-destrutiva, muito confiável e permite a determinação direta e rápida de várias propriedades, sem necessidade do pré-tratamento da amostra [3]. A espectroscopia de infravermelho inclui muitos tipos diferentes de equipamentos que operam em regiões diferentes, com diferentes tipos de detectores e acessórios. Espectroscopia de infravermelho médio com transformada de Fourier (FTIR) tornou-se uma das principais técnicas analíticas utilizadas devido a sua qualidade de rastreio, rapidez e custo de análise. FTIR pode ser acoplado com acessórios como reflexão total atenuada (ATR), que permite a análise de uma vasta gama de componentes sólidos ou líquidos [4]. Nos últimos anos, muitos trabalhos têm sido desenvolvidos usando FTIR e espectroscopia no infravermelho próximo (NIR)

Nos últimos anos alguns trabalhos têm utilizado as técnicas FTIR e NIR associadas à análise multivariada [4-8]. Santos et al. [9] determinaram o teor de enxofre, assim como algumas propriedades físico-químicas do diesel utilizando a calibração multivariada por mínimos quadrados parciais (PLS). Como valores de referência, foram utilizados os resultados obtidos nos ensaios feitos segundo normas ASTM, para este combustível. No entanto, no próprio trabalho é discutido que os resultados obtidos utilizando as duas técnicas citadas não foram satisfatórios. Foram encontrados coeficientes de correlação de 0,451 e 0,702 para FTIR/PLS.

No presente trabalho apresentamos uma metodologia para determinação do teor de enxofre utilizando dados espectroscópicos de FTIR associados à calibração multivariada por PLS. Os modelos de calibração foram construídos utilizando toda a faixa espectral, regiões do espec- tro e seleção de variáveis pelo método stepwise. Foi utilizado como método de referência a norma ASTM D4294. O objetivo é apresentar uma metodologia simples, de baixo custo e de curto tempo de análise para determinar o teor de enxofre no combustível. Diferente dos resultados obtidos por Santos et al., os resultados aqui apresentados mostram modelos com altos coeficientes de correlação e baixos erros de previsão.

\section{Material e métodos}

\subsection{Amostras}

As amostras de diesel foram cedidas pelo Laboratório de Análises de Combustíveis (LEC), da Universidade Federal de Minas Gerais, Brasil. O teor de enxofre das amostras foi determinado segundo norma ASTM D4294, utilizando um espectrômetro de fluorescência de raios-X por energia dispersiva, modelo Shimadzu EDX 800.

\subsection{ATR-FTIR analysis}

Os espectros de ATR-FTIR foram obtidos usando um espectrômetro de infravermelho ABB Bomen modelo MB 102 equipado com acessório de ATR e detector de sulfato de glicerina deuterada. Os espectros foram coletados a temperatura de $16 \pm 1{ }^{\circ} \mathrm{C}$, com média de 32 varreduras e resolução de $2 \mathrm{~cm}^{-1}$. A limpeza do acessório de ATR foi feita com acetona. Os dados foram tratados quimiometricamente usando o software MINITAB ${ }^{\circledR}$, version 14.

\subsection{Tratamento quimiométrico}

A análise dos dados foi realizada utilizando-se o software estatístico Minitab, version 14. Este software possui uma limitação operacional quanto ao número de variáveis (1000 variáveis) a 
serem processadas para um conjunto completo de dados (conjunto de treinamento e validação). Nosso conjunto total de dados perfazia 1542 variáveis (771 variáveis tanto para o conjunto de treinamento quanto para o de validação). Esta limitação nos impede de calcular os valores da raiz quadrada do erro médio de predição (RMSEP), ou seja, não é possível validar o modelo. Para resolver essa limitação, foram adotados dois procedimentos: o primeiro procedimento foi utilizar uma ferramenta de seleção de variáveis disponível no próprio software. Assim, podemos selecionar aquelas variáveis mais representativas utilizando o método stepwise. Soares et al. [4] utilizaram a seleção de variáveis stepwise para determinação de adulteração em biodiesel. A maior vantagem é a simplicidade do método [1]. O segundo procedimento foi selecionar algumas regiões específicas da faixa espectral. Essas regiões foram selecionadas segundo a importância das variáveis preditoras, a partir do desenvolvimento de um primeiro modelo construído com todas as variáveis.

\section{Resultados e discussão}

\subsection{Análises de ATR-FTIR}

O enxofre está presente no diesel em uma concentração muito pequena, estando sempre associado a cadeias carbônicas ou na forma de sulfetos, o que é um problema para a sua quantificação. Por outro lado, quando pensamos no seu efeito acumulativo com o tempo na atmosfera estes pequenos teores tornam expressivos. No caso das amostras utilizadas nesse trabalho a concentração variou de 400 a $2500 \mathrm{mg} \cdot \mathrm{kg}^{-1}$.

Na Figura 1 é mostrado o espectro de uma das amostras de diesel utilizadas no trabalho. A banda de absorção em $2955 \mathrm{~cm}^{-1}$ pode ser atribuída à deformação axial assimétrica de grupos metila, e a banda de absorção em $2930 \mathrm{~cm}^{-1}$ pode ser atribuída a deformação axial assimétrica de grupos metileno. Absorção por volta de $1455 \mathrm{~cm}$
${ }^{-1}$ pode ser atribuída à deformação axial $\mathrm{C}=\mathrm{C}$ de anel aromático [10].

O enxofre está presente no diesel em cadeias carbônicas ou como sulfetos. Em ambos os casos as bandas de absorção no espectro FTIR são muito fracas, e em conseqüência, praticamente não são percebidas visualmente. Hipler et al. [11] estudando compostos de enxofre identificou freqüências correspondentes à transição vibracional da ligação C-S entre 530 e $975 \mathrm{~cm}^{-1}$. Na Figura 1, podemos ver o quanto é difícil de observar estas bandas de absorção. 


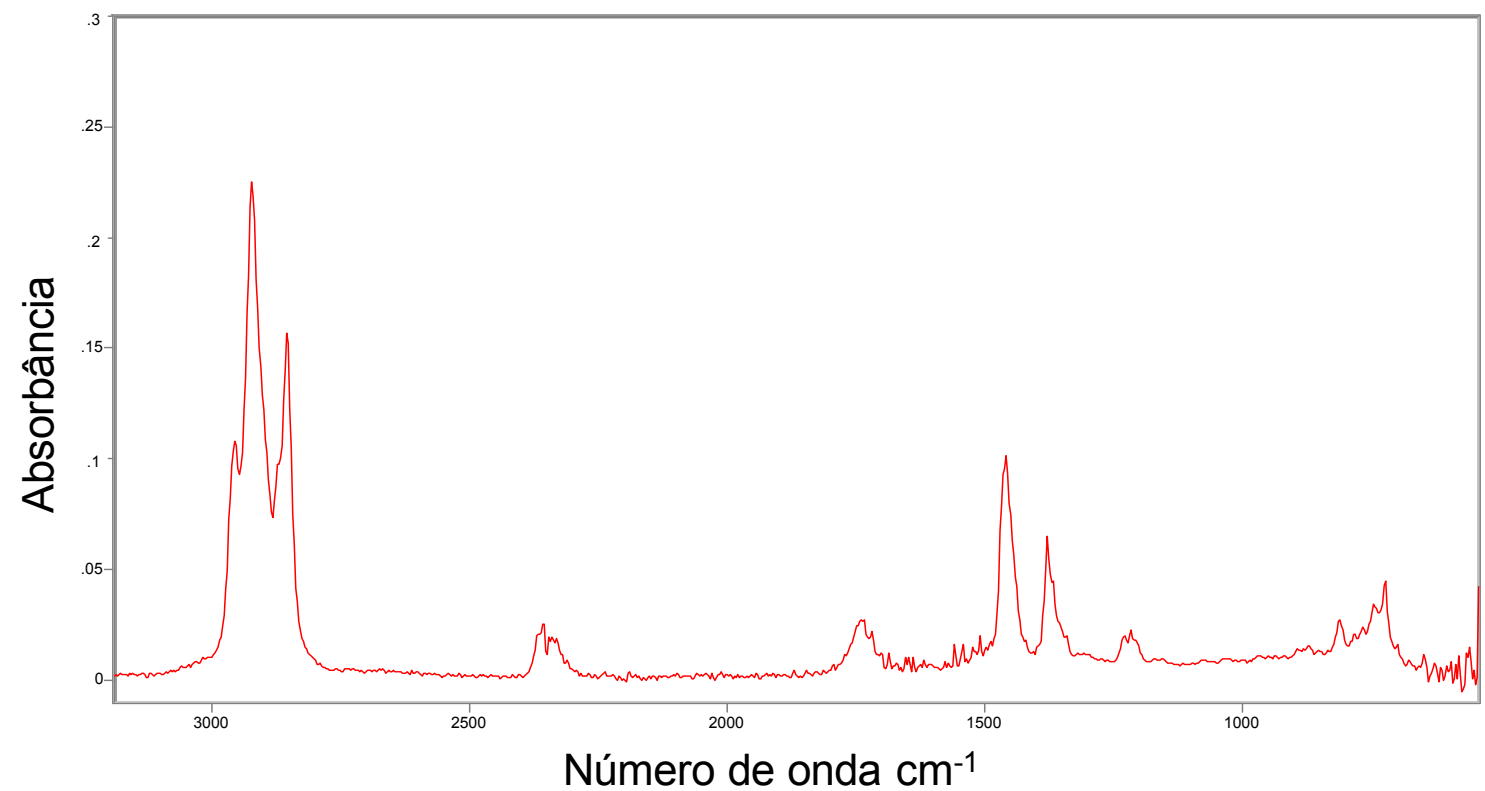

Figura 1. Espectro de infravermelho de uma amostra de diesel

\subsection{Tratamento quimiométrico}

Com os dados obtidos dos espectros de FTIR foram construídos quatro modelos PLS: o modelo a foi construído com todas as variáveis. Os modelos b e c foram construídos a partir de informações relativas aos valores dos preditores encontrados no modelo a. O modelo b (região 1) foi construído com os valores de preditores de 1 a $99\left(665-856 \mathrm{~cm}^{-1}\right)$ e $251-570\left(1145\right.$ e $\left.2717 \mathrm{~cm}^{-1}\right)$. O modelo c (região 2) construído com os valores de preditores de 571 a $771\left(2713-3099 \mathrm{~cm}^{-1}\right) \mathrm{O}$ modelo d foi construído utilizando o método de seleção de variáveis stepwise. 665 e 856 cm-1 e 1145 e $2717 \mathrm{~cm}^{-1}$.

A Figura 2 mostra o resultado da calibração PLS (valores reais versus valores preditos) para os quatro modelos. Observa-se que os quatro modelos são bastante semelhantes. Na Figura 2a são destacadas três regiões. Essas regiões dizem respeito aos teores de enxofre estabelecidos pela legislação brasileira em março de $2006^{\dagger}$, e correspondem aos limites máximos de enxofre por região: 500 mg.kg-1, 2000 mg.kg-1 e 3500 mg.kg-1. Esses limites máximos foram fixados considerando-se o fluxo de veículos por região no país. $\mathrm{Na}$ região cujo limite máximo é de $500 \mathrm{mg} . \mathrm{kg}^{-1}$, observa-se que algumas amostras estão em desacordo com a legislação, pois ultrapassaram esse limite. Já na região cujo limite máximo é de $3500 \mathrm{mg} \cdot \mathrm{kg}^{-1}$, os valores estiveram bem abaixo do limite. 
a)

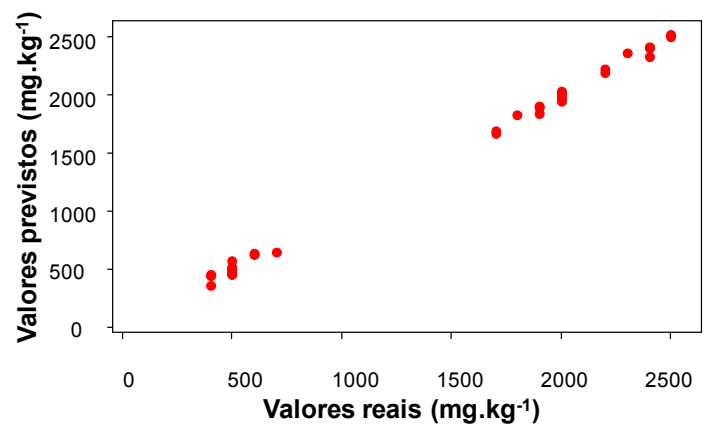

c)

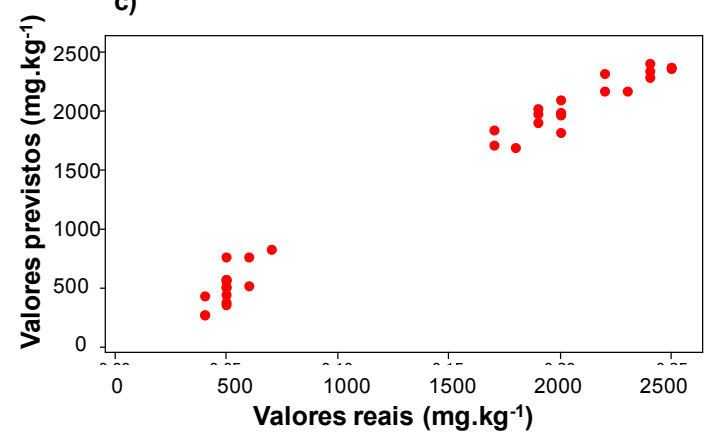

b)

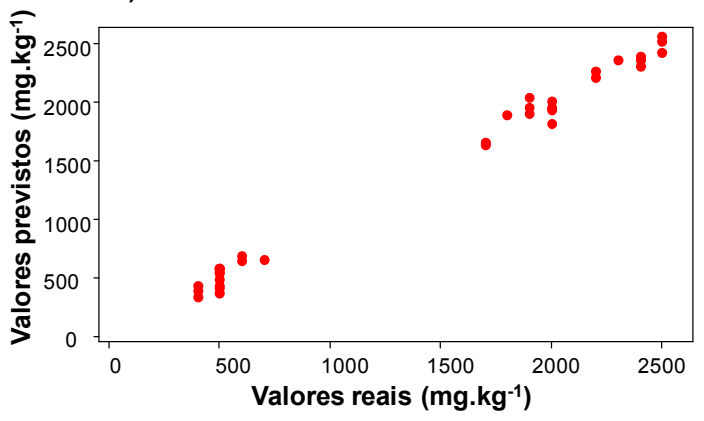

d)

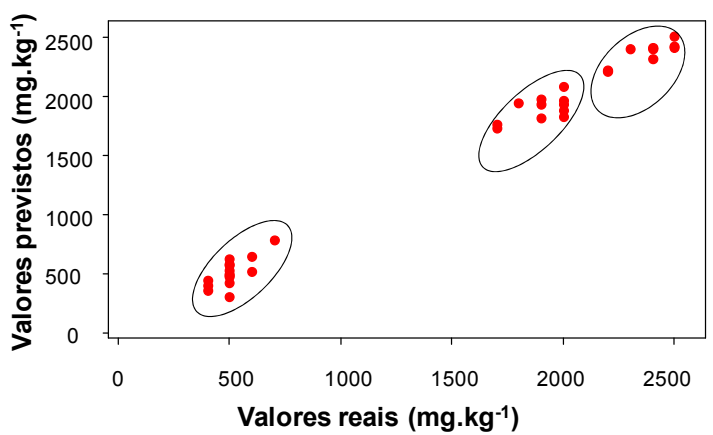

Figura 2. Valores reais e valores preditos na calibração PLS para os modelos: a) completo, b) região 1, c) região 2 e d) sem seleção de variáveis

A Figura 3 mostra à resposta das variáveis preditoras do modelo a (construído com todas variáveis) para as amostras analisadas. Observa-se na Figura 3 que os valores preditores de 100 a 250 podem ser retirados da matriz de dados, pois não fornecem informação relevante para o cálculo da concentração de enxofre. Os valores de preditores de 570 a 771 apresentam respostas com grande discrepância entre grupos de amostras, diferentemente dos demais valores de preditores do espectro. Assim estes, valores foram considerados separadamente para construção do modelo c. 


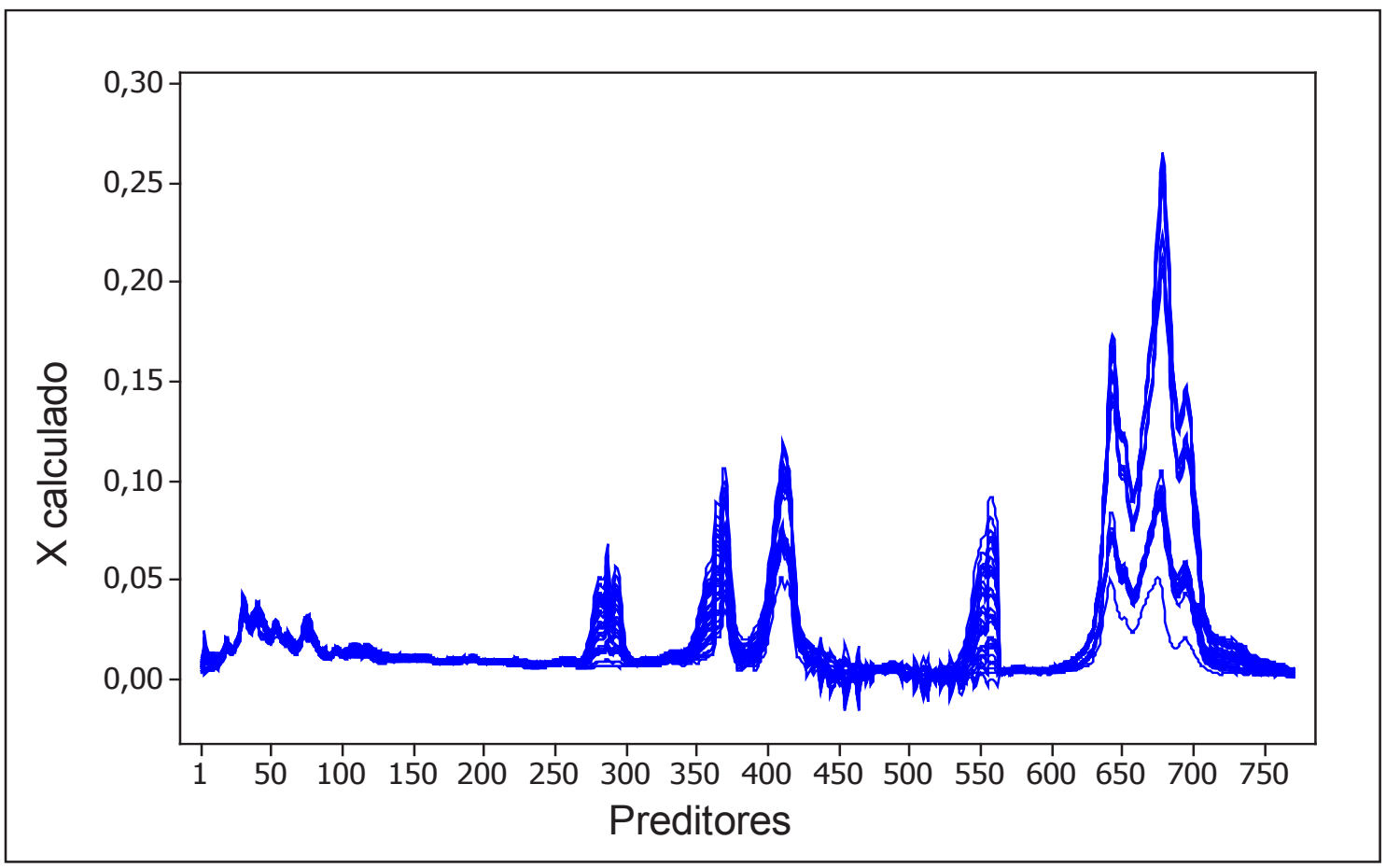

Figure 3. Respostas dos valores de preditores no modelo a

A Figura 4 apresenta os coeficientes das variáveis preditoras selecionadas pelo método stepwise. Nesta Figura são destacadas as variáveis que apresentaram os maiores coeficientes, ou seja, aquelas que são as mais significativas para a construção do modelo de calibração. A maior parte das variáveis com maiores coeficientes se encontra na região de impressão digital (abaixo de $1000 \mathrm{~cm}^{-1}$ ). As variáveis 759 , 869 e $962 \mathrm{~cm}^{-1}$ apresentaram coeficientes positivos. Isso significa que quanto maior a absorção nessas variáveis maior será o teor de enxofre. Já as variáveis 765, 941 e $2746 \mathrm{~cm}^{-1}$ apresentaram coeficientes negativos. Em conseqüência, quanto maior a absorção nessas variáveis menores deverão ser os teores de enxofre preditos. 


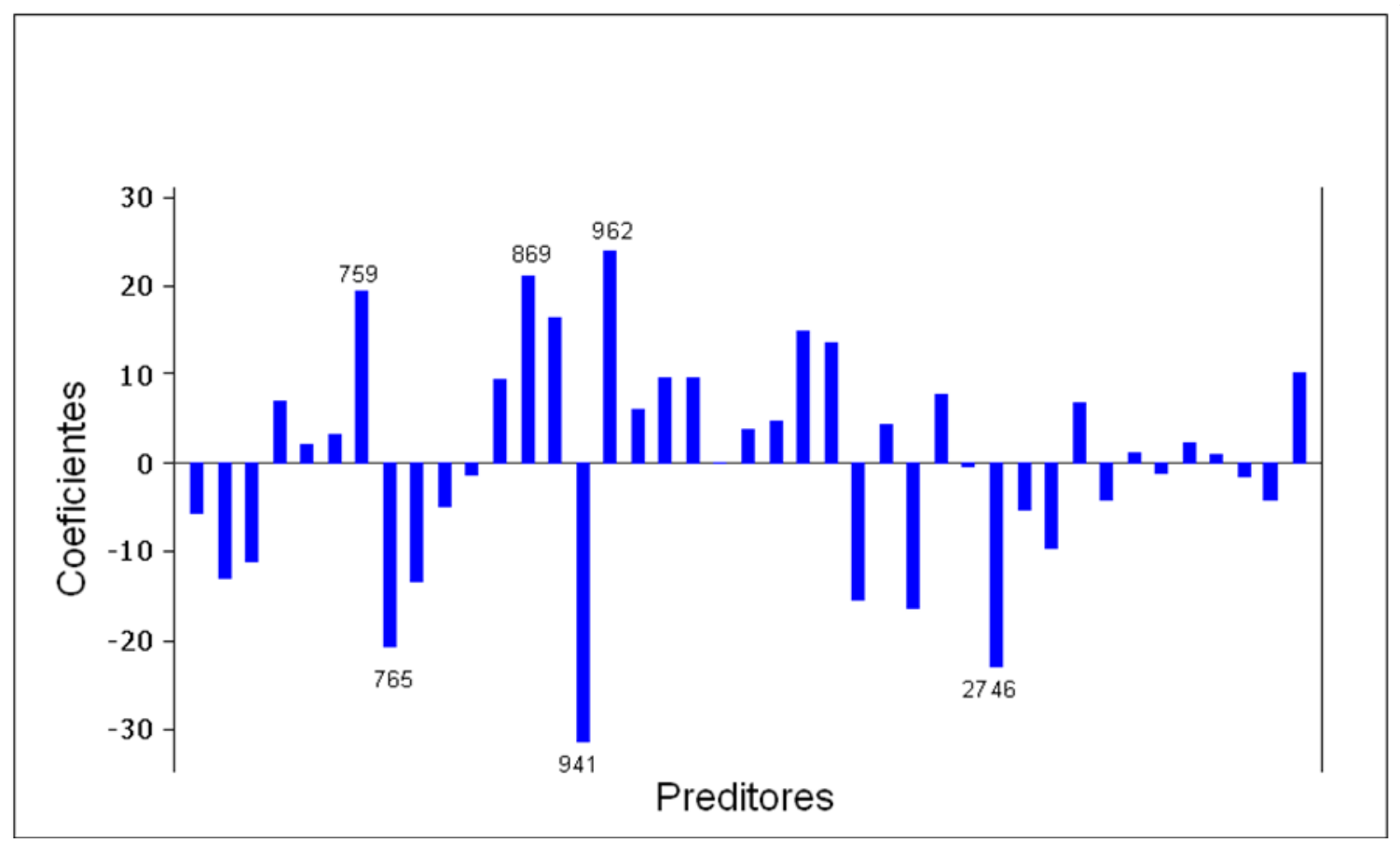

Figura 4 . Coeficientes das variáveis preditoras selecionadas pelo método stepwise

A Tabela 1 apresenta os valores obtidos para o coeficiente de correlação (R), a raiz quadrada do erro médio de calibração (RMSEC), a raiz quadrada do erro médio de previsão (RMSEP), variáveis latentes (LV) e sensibilidade (SEN), para os modelos de calibração PLS construídos. Para os modelos a, b e d foram encontrados os valores de RMSEC 0,004, 0,007, e 0,007, respectivamente. Podendo ser considerados, dessa forma, de eficiência semelhante. O modelo c apresentou eficiência menor que os modelos anteriores, com RMSEC 0,01 . No modelo c, o coeficiente de correlação e a sensibilidade foram menores que os demais modelos e o RMSEC e RMSEP foram maiores. Isso significa que a faixa espectral utilizada no modelo c não deve ser utilizada na predição do teor de enxofre, porque leva a erros de predição maiores. Dessa forma, entre os quatro modelos desenvolvidos, os modelos b e d apresentaram os melhores resultados; maior concordância entre valor real e predito (menores valores de RMSEC), maior sensibilidade e por serem validados, o que não foi possível de fazer com o modelo construído com todas as variáveis.

Tabela 1. Resultados da calibração PLS

\begin{tabular}{cccccc}
\hline Modelo & R & RMSEC & RMSEP & LV & SEN \\
\hline a- completo & 0.997 & 0.004 & $*$ & 8 & $3.41 \times 10^{-2}$ \\
b- região 1** & 0.992 & 0.007 & 0.038 & 8 & $3.22 \times 10^{-2}$ \\
c- região 2*** & 0.982 & 0.01 & 0.16 & 8 & $8.55 \times 10^{-3}$ \\
d- stepwise & 0.990 & 0.007 & 0.028 & 8 & $1.31 \times 10^{-2}$ \\
\hline
\end{tabular}

*Número de variáveis excede a capacidade de processamento do software

** Região de 665-856 e 1145 e $2717 \mathrm{~cm}^{-1}$

*** Região de 2713-3099 $\mathrm{cm}^{-1}$ 
Para verificar a eficiência dos modelos b e d foi aplicado o teste-f, considerando, para tanto, os valores de sensibilidade. Os resultados são apresentados na Tabela 2. Observa-se na Tabela 2 que o valor encontrado no teste-f é maior que o f tabelado. Podemos inferir com $95 \%$ de confiança que a diferença entre os dois modelos é estatisticamente significante.

Tabela 2. Teste-f para comparar os modelos

\begin{tabular}{ccc}
\hline Modelos & Teste-f & f-tabelado \\
\hline b e d & 2.66 & 2.43 \\
\hline
\end{tabular}

Considerando o conjunto de amostras utilizado e o resultado obtido no teste-f, pode se considerar que o melhor modelo para determinar o teor de enxofre é o modelo com faixa espectral compreendida de $665-856$ e $1145-2717 \mathrm{~cm}^{-1}$.

\section{Conclusões}

A associação entre a técnica de FTIR e a calibração multivariada, PLS mostrou ser uma ferramenta bastante poderosa na determinação do teor de enxofre em amostras de diesel na faixa de concentração estudada (400 a $2500 \mathrm{mg} \cdot \mathrm{kg}^{1}$ ). Os valores obtidos para os modelos desenvolvidos mostraram uma grande concordância entre valores reais e os valores preditos Isto é observado quando analisamos os valores de R, RMSEC e RMSEP, Estes resultados mostraram-se promissores quando comparado àqueles obtidos por Santos et al.

A utilização do método de seleção de variáveis e da seleção de regiões do FTIR para construção dos modelos se mostraram interessantes, pois possibilitaram a validação dos modelos contornando a limitação do software.

$\mathrm{O}$ modelo $\mathbf{b}$ foi o mais eficiente, porque embora apresentando erros de predição, e coeficientes de correlação semelhantes ao modelo d, o modelo $b$ apresentou maior sensibilidade.

\section{Referências}

[1] J. Mulrroney, J Clifford, C. Fitzpatrick, E. Lewis, Sensors and Actuators A, (136) (2007) 104.

[2] A. Marafi, A. Al-Hindi, A. Stanislaus, Fuel Processing Technology, (88) (2007) 905.

[3] M.F. Pimentel, L. S. G.Teixeira, G. M. S. Ribeiro, R. S. Cruz, L. Stragevitch, J. G. A. P. Filho, Microchemical Journal, (82) (2006) 201

[4] I.P. Soares, T.F. Rezende, R.C. Silva, E.V.R. Castro, I.C.P. Fortes, Energy \& Fuels, (22) (2008) 2079.
[5] R.C.C. Pereira, V.L. Skrobot, E.V.R. Castro, I.C. P. Fortes, V. M. D. Pasa, Energy \& Fuels, (20) (2006) 1097.

[6] G. Knothe, J. Am. Oil Chem. Soc., (78) (2001) 1025.

[7] J.S.Oliveira, R. Montalvão, L. Daher, A.Z. Suarez, J.C. Rubim, Talanta, (69) (2006) 1278.

[8] Y. B. Che Man, G. Setiowaty, Food Chem., (66) (1999)109.

[9] V.O. Santos Jr., F.C.C. Oliveira, D.G. Lima, A.C. Petry, E. Garcia, P.A.Z. Suarez, J.C. Rubim, Analytica Chimica Acta, (547) (2005) 188

[10] R. M. Silverstein, F. X. Webster, Spectrometric Identification of Organic Compounds, $6^{\text {th }}$ ed.; Wiley: New York, 1998.

[11] F. Hipler, Fischer, R.A. M. Winter, Journal molecular structure, 2003, 181-183 